\title{
Broadband Class-E Power Amplifier for Space Radar Application
}

\author{
T. Quach*, P. Watson*, W. Okamura**, E. Kaneshiro**, A. Gutierrez-Aitken**, T. Block**, \\ J. Eldredge**, T. Jenkins*, L. Kehias*, A. Oki**, D. Sawdai**, R. Welch*, and R. Worley* \\ * Air Force Research Laboratory, 2241 Avionics Circle, BLDG 620, Wright-Patterson AFB, \\ Ohio 45433
}

** TRW Electronics and Technology Division, One Space Park, Redondo Beach, CA 90278

\begin{abstract}
We report on a broadband high efficiency power amplifier using Indium Phosphide (InP) double heterojunction bipolar transistor (DHBT) technology. The amplifier demonstrated a record bandwidth for a Class-E power amplifier at X-Band. This circuit achieved 49-58\% PAE, 18.5-23.9 dBm output power, and 9.6-10.5 dB gain across 9-11 GHz.
\end{abstract}

\section{Introduction}

Modern space-based radar systems for military applications place new requirements on RF power amplifiers due to the desire to reduce system size, weight, and launch cost. A major shift in amplifier specifications focuses more on circuit efficiency to reduce prime power requirements and possibly improve system reliability due to lower power dissipation. Furthermore, broadband high efficiency circuits will enable enhanced system capability. As shown in [1], switch mode amplifier design, such as the Class-E power amplifier, can potentially achieve $100 \%$ drain or collector efficiency when driven into deep saturation. However, most demonstrations of Class-E amplifiers have been at low RF frequency due to device limitations. Recent demonstrations of Class-E power amplifiers at X-Band were reported by [2] and [3] with limited bandwidth. This paper describes a broader bandwidth circuit that utilizes the low loss transmission line approach by [4] and [5] to demonstrate a Class-E power amplifier at X-Band with $20 \%$ bandwidth.

\section{DHBT Device Characteristics and Test Circuit Results}

The X-Band power amplifier employs a DHBT device with the material structure of InAlAs/InGaAs/InP (emitter/base/collector) grown by molecular beam epitaxy on a 3 -inch Indium Phosphide substrate. The device consists of $1.5 \mu \mathrm{m} \mathrm{X}$ $30 \mu \mathrm{m} \times 4$ fingers totaling $180 \mu \mathrm{m}^{2}$ emitter area (Fig. 1). For this unit cell, the offset voltage, maximum collector current, and the breakdown voltage $\left(\mathrm{BV}_{\mathrm{ceo}}\right)$ are about $50 \mathrm{mV}, 200 \mathrm{~mA}$, and $18 \mathrm{~V}$, respectively [6]. The combination of low offset voltage, high breakdown voltage, and high gain makes the InP DHBT an attractive technology for high efficiency performance.

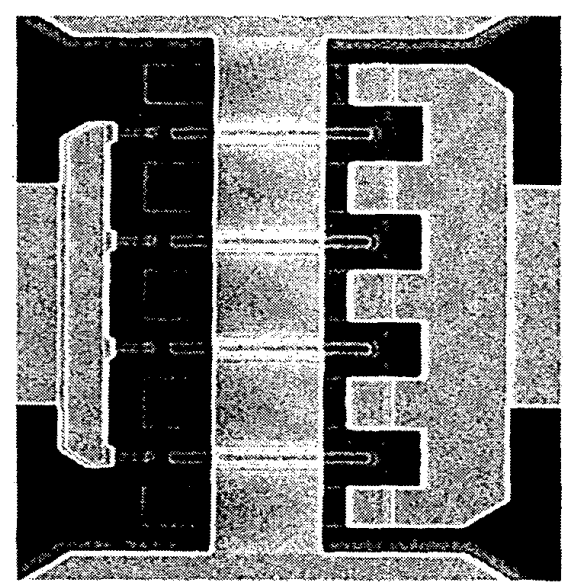

Figure1: SEM Photograph of the four-finger DHBT device. Each finger is $1.5 \mu \mathrm{m} \mathrm{X} 30 \mu \mathrm{m}$ totaling $180 \mu \mathrm{m}^{2}$ of emitter area.

A modified Gummel-Poon based model has been used to simulate large signal performance of the DHBT device. Model parameters were fitted to measured data across various bias conditions. As a result, the model appears to capture large signal performance with good accuracy [7]. This model is used to simulate the ideal case where tuner models are used to terminate harmonics and also provide the optimal load condition for peak efficiency performance. Simulations suggest, that under ideal conditions, the DHBT device can achieve approximately $73 \%$ PAE, $22.3 \mathrm{dBm}$ output power, and $12.3 \mathrm{~dB}$ gain at $10 \mathrm{GHz}$. Fig. 2 gives PAE contours for various fundamental load impedances. This plot also indicates that if device harmonics are properly terminated with lossless input and output networks, the 
DHBT has a wide range of load impedances for which high efficiency performance is possible.

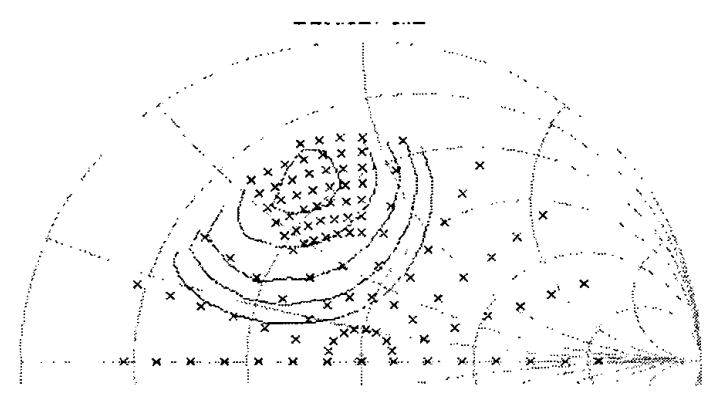

Figure 2: Power-added-efficiency contours of the DHBT device based on ideal harmonic terminations and fundamental matching. This plot shows five PAE contours with a $4 \%$ step ranging from $73-57 \%$ PAE. The optimal impedance is located at $22+\mathrm{j} 34 \Omega$.

To verify the model performance for Class-E operation, the test circuit shown in Fig. 3 was designed and fabricated. This circuit is a single stage design for $10 \mathrm{GHz}$ operation. The output network of the circuit provides an open at the second harmonic and the fundamental matching condition at the device output terminal. The DHBT device is externally biased through a set of Bias-Tees at the input and output terminals.

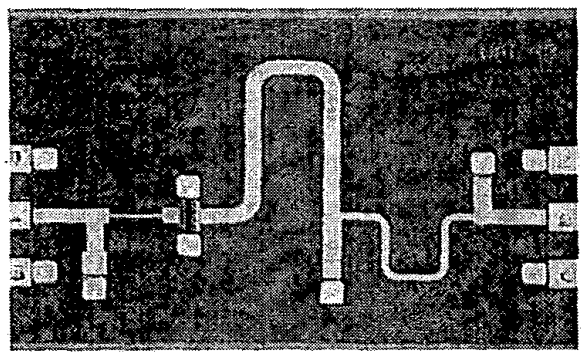

Figure 3: Photograph of the test circuit. Circuit dimension is $2 \mathrm{~mm} \mathrm{X} 1.3 \mathrm{~mm}$.

Fig. 4 compares the circuit's simulated and measured PAE, output power, and gain. This circuit was tested on a load-pull setup with both input and output tuners set to $50 \Omega$. Bias conditions for power characterization were set to $\mathrm{Vce}=4 \mathrm{~V}$ and Vbe $=0.7 \mathrm{~V}$. The best PAE was recorded at $10 \mathrm{dBm}$ input drive yielding $55.9 \%$ PAE, $20.5 \mathrm{dBm}$ output power, and $10.5 \mathrm{~dB}$ gain.

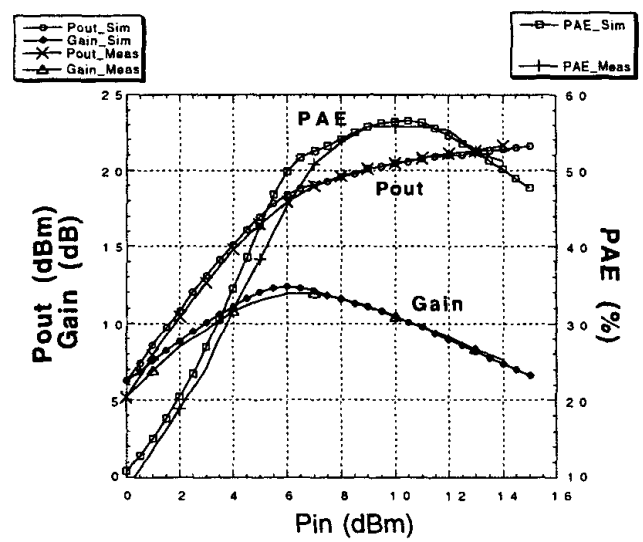

Figure 4: Simulated vs. measured data for the single stage test circuit at $10 \mathrm{GHz}$. The device is biased at $\mathrm{Vce}=4 \mathrm{~V}, \mathrm{Vbe}=0.7 \mathrm{~V}$, and $\mathrm{Ic}=44 \mathrm{~mA}$.

\section{Broadband Power Amplifier Results}

The broadband high efficiency power amplifier is a single stage design shown in Figure 5 . The output network design is a compromise between having a low insertion loss network and meeting the necessary requirements for the Class-E design. This is accomplished by using two open stubs in conjunction with a shunt capacitor. The first shunt stub presents high impedances to second harmonics (18-22 GHz) at the device output. The combination of the second shunt stub and the shunt capacitor present low impedances at third harmonics $(27-33 \mathrm{GHz})$ and also transform to the load impedances at fundamental for high efficiency performance. This is accomplished without affecting circuit tuning at second harmonics. This design simplifies the output-matching network to minimize network insertion loss. The input network consists of low pass LC network to provide a good input match.

On-wafer small-signal measurements are plotted in Fig. 6. Measurements were carried out at Vce $=4 \mathrm{~V}$ and $\mathrm{Vbe}=0.7 \mathrm{~V}$. Measured data show input and output VSWR are approximately $2: 1$ and small signal gain ranges from 12-14 $\mathrm{dB}$ across the design frequency of 9-11 GHz. Furthermore, the $S_{21}$ plot reflects the effects of the broadband second harmonic termination in that $\mathrm{S}_{21}$ is below $-30 \mathrm{~dB}$ from $18-22 \mathrm{GHz}$. This broadband harmonic termination enables high efficiency operation. Power characterization of the circuit was taken on a load-pull test station with input and output tuners set to $50 \Omega$ and biased at $\mathrm{Vce}=4.5 \mathrm{~V}$ and $\mathrm{Vbe}=0.6 \mathrm{~V}$. Fig. 7-9 are plots of measured PAE, output power, and gain for $9 \mathrm{GHz}, 10 \mathrm{GHz}$, and $11 \mathrm{GHz}$, respectively. Power 
measurements show the circuit achieved 49-58\% PAE, 18.5-23.9 dBm output power, and 9.6-10.5 $\mathrm{dB}$ gain across $9-11 \mathrm{GHz}$.

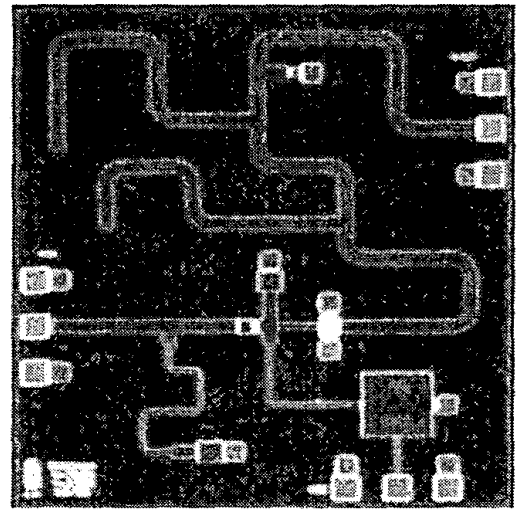

Figure 5: Photograph of the broadband Class-E power amplifier. Circuit dimension is $2 \mathrm{~mm} \mathrm{X}$ $2.2 \mathrm{~mm}$.

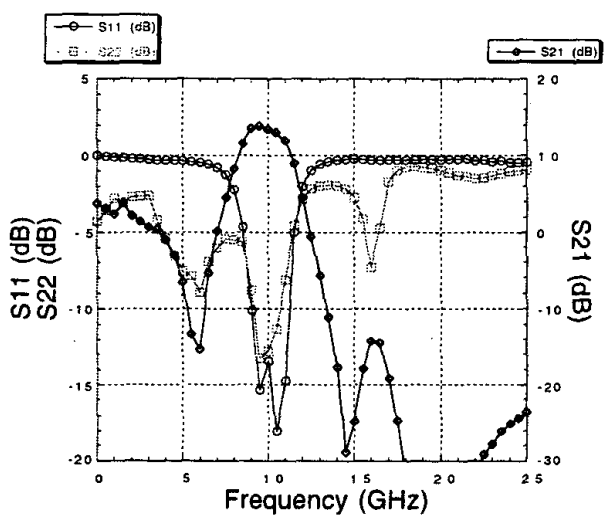

Figure 6: S-parameter measurements of the power amplifier at $\mathrm{Vce}=4 \mathrm{~V}, \mathrm{Vbe}=0.7 \mathrm{~V}$, and $\mathrm{Ic}=37 \mathrm{~mA}$.

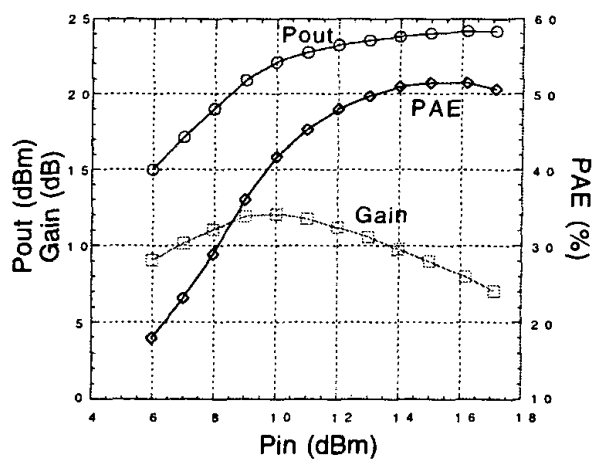

Figure 7: Measured output power, gain, and PAE at $9 \mathrm{GHz} . \mathrm{Vce}=4.5 \mathrm{~V}$ and $\mathrm{Vbe}=0.6 \mathrm{~V}$

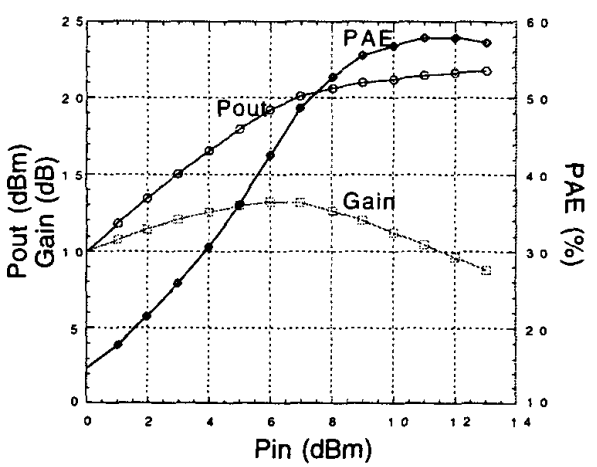

Figure 8: Measured output power, gain, and PAE at $10 \mathrm{GHz} . \mathrm{Vce}=4.5 \mathrm{~V}$ and $\mathrm{Vbe}=0.6 \mathrm{~V}$.

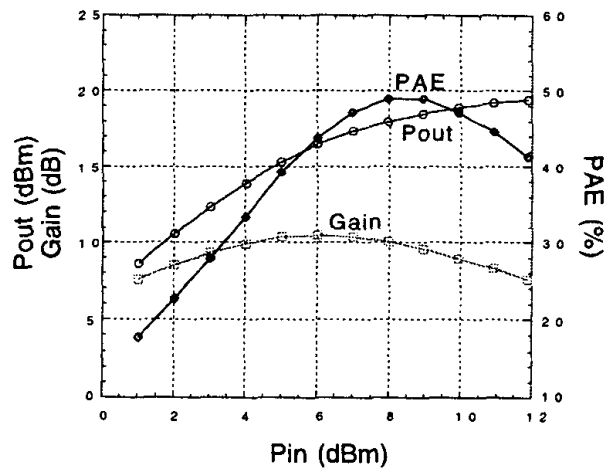

Figure 9: Measured output power, gain, and PAE at $11 \mathrm{GHz} . \mathrm{Vce}=4.5 \mathrm{~V}$ and $\mathrm{Vbe}=0.6 \mathrm{~V}$ 


\section{Conclusion}

The InP DHBT device was analyzed using nonlinear simulation with a modified Gummel-Poon model. Fundamental and harmonic terminations of the device were tuned for peak efficiency. This analysis gives a theoretical efficiency limitation of the DHBT device for a given material structure. For this ideal case, simulations suggest the DHBT device is capable of reaching $73 \%$ PAE. A single stage test circuit was used to evaluate the large signal model. Measured and simulated results show excellent agreement in PAE, output power, and gain. Finally, a broadband high-efficiency power amplifier has been demonstrated by the low-loss transmission line approach. The Class-E power amplifier achieved 49$58 \%$ PAE, 18.5-24.0 dBm output power, and 9-10.5 $\mathrm{dB}$ gain across $9-11 \mathrm{GHz}$. To the best of our knowledge, this circuit achieved the widest bandwidth for a switch mode design at X-Band.

\section{Acknowledgements}

The authors would like to thank T. Kemerly, K. Nakano, M. Calcatera, and R. Kagiwada for their support. The authors further acknowledge the Air Force Dual Use Science \& Technology Office for its support under Cooperative Agreement No. F3361599-2-1471.

\section{References}

[1] N.O. Sokal and A.D. Sokal, "Class E - A New Class of High-Efficiency Tuned Single - Ended Switching Power Amplifiers, “ IEEE J. of Solid-State Circuits, Vol. SC-10, No. 3, pp. 168-176, June 1975.

[2] M. Weiss and Z. Popovic, "A $10 \mathrm{GHz}$ High Efficiency Active Antenna," 1999 IEEE Microwave Theory and Tech. Int. Microwave Symp. Digest, pp. 663-666, June 1999.

[3] T. Quach, W. Okamura, A. Gutierrez-Aitken, T. Jenkins, E. Kaneshiro, L. Kehias, A. Oki, D. Sawdai, P. Watson, R. Welch, R. Worley, and H.C. Yen, "UltraEfficient X-Band and Linear Efficient Ka-Band Power Amplifiers using Indium Phosphide Double Heterojunction Bipolar Transistors," 2001 International Conference on Indium Phosphide and Related Materials., pp. 501-504, May 2001.

[4] T. Mader and Z. Popovic, "The Transmission Line High Efficiency Class-E Amplifier," IEEE Microwave and Guided Wave Lett., Vol.5, pp. 290-292, Sept. 1995.

[5] A. Wilkinson and J. Everard, "Transmission Line Load Network Topology for Class-E Power Amplifiers," IEEE Transactions on Microwave Theory and Techniques, pp. 1202-1210, June 2001.

[6] K.W. Kobayashi, A.K. Oki, A. Gutierrez-Aitken, P.Chin, L.T. Tran, Li-W Yang, D. Sawdai, E. Kaneshiro, P.C. Grossman, K. Sato, T.R. Block, H.C. Yen, and D.C. Streit, "InP Heterojunction Bipolar Transistor (HBT) Technology for Power Applications," Electro-Chemical Society Conference, Toronto, Canada, May 2000.

[7] P. C. Grossman and J. Choma, "Large Signal Modeling of HBT's Including Self-Heating and Transit Time Effects," IEEE Transactions on Microwave Theory and Techniques, Vol. 40, No.3, pp. 449-464, March 1992 